\title{
(RE)PENSAR OS CONFLITOS INTRAGRUPAIS: DESEMPENHO E NÍVEIS DE DESENVOLVIMENTO ${ }^{1}$
}

\author{
Isabel Cristina Dórdio Dimas ${ }^{2}$ \\ Paulo Renato Lourenço ${ }^{3}$ \\ José Miguez ${ }^{4}$
}

Resumo: Contrariando as abordagens tradicionais do conflito intragrupal, que consideram este fenómeno negativo para o desempenho, as perspectivas mais recentes sublinham as suas potencialidades ao nível da inovação e da criatividade das equipas de trabalho. Os estudos empíricos desenvolvidos têm, no entanto, encontrado poucas evidências para a funcionalidade do conflito.

O presente artigo procura contribuir para uma melhor compreensão das relações entre o conflito, as estratégias de gestão de conflitos e o desempenho grupal, por um lado, bem como da forma como os conflitos são vividos e geridos ao longo do desenvolvimento grupal, por outro. Participaram no nosso estudo 68 equipas de trabalho do departamento de produção de 8 organizações do sector industrial que desempenhavam tarefas com um baixo nível de complexidade. Os resultados revêlaram que o conflito centrado no desenvolvimento das tarefas se relaciona negativamente com o desempenho e que a utilização de estratégias integrativas se assume como a única abordagem aos conflitos com um impacto positivo nos resultados das equipas de trabalho. As análises realizadas permitiram também concluir que a emergência de conflitos é diferente, ao longo do desenvolvimento grupal.

Para testar o padrão de resultados encontrado, importa realizar novos estudos com equipas cujas tarefas requeiram uma elevada coordenação de esforços e remetam para resultados caracterizados por uma elevada imprevisibilidade.

Palavras-chave: conflito intragrupal, estratégias de gestão de conflitos, desempenho, níveis de desenvolvimento.

\footnotetext{
${ }^{1}$ A correspondência relativa a este artigo deve ser endereçada a Isabel Cristina Dórdio Dimas, Faculdade de Psicologia e de Ciências da Educação da Universidade de Coimbra, e-mail: idimas@mail.fpce.uc.pt.

Os autores gostariam de agradecer às empresas que colaboraram na presente investigação.

Este estudo foi financiado pela Fundação para a Ciência e Tecnologia, através do Programa Operacional Ciência e Inovação 2010 (n. ${ }^{\circ}$ da bolsa: SFRH/BD/16172/2004).

${ }^{2}$ Faculdade de Psicologia e de Ciências da Educação da Universidade de Coimbra.

${ }^{3}$ Faculdade de Psicologia e de Ciências da Educação da Universidade de Coimbra, Coimbra.

${ }^{4}$ Faculdade de Psicologia e de Ciências da Educação e Escola de Gestão do Porto, Universidade do Porto, Porto.
} 
(Re)Thinking intragroup conflict: performance and group development (Abstract): Against the traditional perspective of intragroup conflict, that viewed this phenomenon as negative to group performance, the recent approaches argue that conflict stimulates creativity and the innovation of workgroups. Nonetheless, the empirical studies developed didn't find support to the positive effect of conflict in-group functioning.

In this paper we try to contribute to a better understand of the relationships between conflict, handling conflict strategies and group development. We analyze, also, the way conflicts are lived and managed along the group temporal moments. Sixty-eight workgroups from the production department of eight industrial companies agreed to participate in this study. All workgroups performed non-complex tasks.

Results revealed that task conflict is negatively related with performance and that integrating is the only strategy of handling conflict with a positive effect on the workgroups results. Our analysis suggests also that the emergence of intragroup conflict is different along the group temporal moments.

Other studies are necessary with teams that performed complex and non routine tasks to test the pattern of results found.

Key-words: intragroup conflict, conflict management, performance, group development.

\section{Introdução}

Nos últimos anos, o grupo tem vindo a constituir-se como a unidade de trabalho privilegiada nas organizações, colocando novos desafios à gestão das pessoas (Guzzo \& Shea, 1992). A interacção e interdependência que caracterizam os grupos organizacionais geram tendências para a acção e constituem uma fonte potencial de conflito. Realidade incontornável nas organizações, o conflito intragrupal tem sido associado quer a aspectos positivos, como o aumento dos níveis de inovação e de criatividade (Tjosvold, 1997), quer a aspectos negativos, nomeadamente à redução da coesão grupal, à diminuição dos níveis de desempenho e ao aumento do turnover (Spector \& Jex, 1998). A perspectiva dominante na literatura da especialidade sublinha as vantagens da existência de um determinado nível de conflito nos grupos de trabalho, desde que centrado em questões relativas ao desenvolvimento das tarefas (Schweiger \& Sandberg, 1989; Turner \& Pratkanis, 1997). No entanto, as evidências empíricas apontam para resultados contraditórios (De Dreu \& Weingart, 2003b).

Com o presente artigo, procuramos, num primeiro momento, contribuir para uma melhor compreensão das relações que o conflito estabelece com o desempenho grupal, considerando o papel exercido pela forma como, 
no grupo, são geridas as situações conflituais. Tendo presente que um grupo assume particularidades que o diferenciam no tempo, pretendemos, num segundo momento, analisar a intensidade das situações conflituais ao longo do crescimento do grupo, tendo em vista uma melhor compreensão, quer do conflito, quer do próprio desenvolvimento grupal.

\section{Tipo de conflito e resultados das equipas de trabalho}

O conflito pode ser definido como uma divergência de perspectivas, percebida como geradora de tensão por, pelo menos, uma das partes envolvidas numa determinada interacção, e que pode ou não traduzir-se numa incompatibilidade de objectivos (De Dreu \& Weingart, 2003a; Dimas, Lourenço \& Miguez, 2005).

A perspectiva tradicional no estudo dos conflitos considerava este fenómeno prejudicial para o funcionamento dos grupos e atribuía-lhe um conjunto de consequências negativas: redução da coesão grupal, deterioração das redes de comunicação, menor produtividade (Deutsch, 1973; Guetzkkom \& Gyr, 1954; Schwenk \& Cosier, 1993). A investigação centrava-se, então, na procura das causas associadas à emergência de situações conflituais, tendo em vista a sua prevenção/eliminação.

Contrariando esta perspectiva, as abordagens mais recentes sugerem que um nível óptimo de conflito pode maximizar os índices de criatividade e de inovação das equipas de trabalho. É nesta perspectiva que se situam autores como Turner e Pratkanis (1994), ao defenderem que estimular os conflitos em grupos com elevados níveis de coesão facilita o confronto dos vários pontos de vista e a análise das várias alternativas propostas, evitando-se, desta forma, a emergência do "pensamento de grupo" (groupthink). Na mesma linha, Putnam (1997) considera os conflitos como motores de mudança individual e organizacional por estimularem o debate de ideias e contribuírem para o encontrar de soluções criativas para os problemas.

A funcionalidade das situações conflituais, ao nível do desenvolvimento e do desempenho dos grupos de trabalho, vai ser, contudo, condicionada, de acordo com as referidas abordagens, pelo tipo de conflito emergente: conflito de tarefa ou conflito afectivo (Amason, 1996; Jehn, 1994).

O conflito de tarefa engloba situações de tensão vividas no grupo, devido à presença de diferentes perspectivas em relação à execução de uma tarefa. Este tipo de conflito tem vindo a ser associado com uma melhoria na qualidade das decisões grupais (Amason, 1996), com uma maior identificação individual com os resultados do grupo e com o aumento do nível de satisfação dos seus membros (Tjosvold, 1991). Jehn (1995) sublinha, no entanto, que o efeito do conflito de tarefa nos resultados grupais é influenciado pelas características das tarefas desempenhadas pelas equipas de traba- 
1ho. Assim, nas equipas que desempenham tarefas de carácter não rotineiro ${ }^{5}$ e de elevada complexidade, a emergência de diferentes alternativas para a resolução de problemas pode conduzir ao encontrar de soluções mais criativas e eficazes. Quando, pelo contrário, as actividades a empreender são estandardizadas, o confronto de ideias pode ameaçar, mais do que beneficiar, a implementação dos procedimentos associados à realização das tarefas.

O conflito afectivo envolve situações de tensão interpessoal entre os membros do grupo como resultado de diferenças de personalidade. Vários estudos têm salientado as consequências disfuncionais deste tipo de conflito: desvia a energia do grupo da tarefa para a resolução de problemas interpéssoais e reduz a qualidade das relações entre os seus membros, o que produz efeitos negativos, quer no desempenho, quer na satisfação grupal (Amason \& Sapienza, 1997; Rentsch \& Zelno, 2003; Simons \& Peterson, 2000).

O impacto diferencial dos dois tipos de conflito nos resultados das equipas de trabalho, defendido ao nível teórico pelas novas abordagens na área do conflito intragrupal, tem encontrado, no entanto, poucas evidências empíricas.

Numa meta-análise com trinta estudos publicados no período compreendido entre o artigo pioneiro de Jehn (1994) e a Academy of Management Conference, em 2001, De Dreu e Weingart (2003b) concluíram que ambos os tipos de conflito têm consequências negativas, tanto ao nível do desempenho, como ao nível da satisfação grupal, colocando em causa a funcionalidade deste fenómeno para o trabalho em equipa. Os autores verificaram, ainda, que a relação negativa entre o conflito de tarefa e o desempenho grupal é mais forte quando as equipas desempenham tarefas complexas do que quando desempenham tarefas rotineiras, o que contraria as posições defendidas por Jehn (1995), a que nos referimos anteriormente.

A análise de estudos mais recentes aponta para dados semelhantes: com efeito, não é encontrada sustentação empírica para o impacto positivo do conflito de tarefa, quer no desempenho (Passos \& Caetano, 2005), quer no bem-estar, satisfação e desejo de os membros permanecerem na equipa (Medina et al., 2005).

Tendo como base os resultados empíricos encontrados pelos diversos estudos presentes na literatura, predizemos que:

Hipótese 1: O conflito afectivo e o conflito de tarefa relacionam-se negativamente com o desempenho grupal.

\footnotetext{
${ }^{5}$ As tarefas não rotineiras exigem, geralmente, a resolução de problemas complexos em contextos de elevada incerteza. As tarefas rotineiras caracterizam-se por uma baixa variabilidade nos métodos e processos utilizados e por um elevado grau de previsibilidade dos resultados (O' Reilly, Williams \& Barsade, 1998).
} 


\section{O papel das estratégias de gestão de conflitos}

As consequências produzidas pelas situações conflituais ao nível dos resultados das equipas de trabalho (desempenho e satisfação) não é independente, segundo a literatura da especialidade, da forma como as mesmas são geridas pelos membros do grupo (Lovelace, Shapiro \& Weingart, 2001; Simons, Pelled \& Smith, 1999). A um nível intragrupal, as estratégias de gestão de conflitos podem ser definidas como o conjunto de respostas dadas pelos membros do grupo perante a emergência de ideias, opiniões e/ou objectivos divergentes (DeChurch \& Marks, 2001; Pruitt \& Rubin, 1986).

De acordo com os modelos bidimensionais (Blake \& Mouton, 1964; Rahim, 1983; Thomas, 1992), a gestão de conflitos é função da combinação de duas dimensões - preocupação consigo (grau em que o indivíduo procura satisfazer os seus objectivos) e preocupação com os outros (grau em que o indivíduo procura satisfazer os objectivos da outra parte).

Figura 1 -Os Estilos de Gestão de Conflitos.

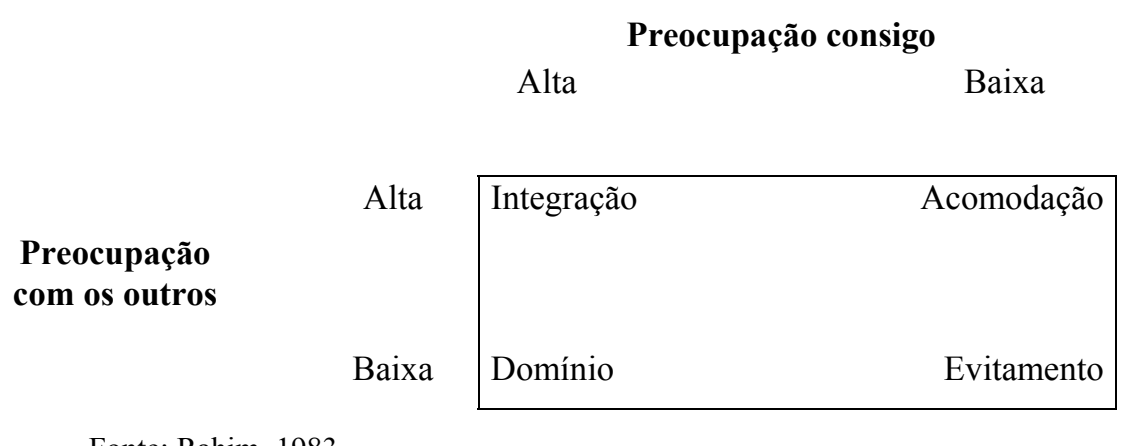

Fonte: Rahim, 1983

Quando existe uma elevada preocupação com os objectivos individuais e com os interesses da outra parte, a estratégia utilizada é a integrãção. A informação é partilhada pelas partes envolvidas, são exploradas de uma maneira construtiva as diferentes formas de pensar um problema e procuram-se soluções que integrem os vários interesses.

A estratégia de acomodação (baixa preocupação consigo e elevada preocupação com os outros) caracteriza-se pelo recurso a concessões unilaterais. A parte envolvida na interacção que adopta esta estratégia cede perante os objectivos da outra parte, negligenciando os interesses individuais.

O domínio (elevada preocupação com os interesses individuais e baixa preocupação com os interesses da outra parte) caracteriza-se pela procura activa de argumentos que sustentem a posição individual, pela manipulação 
das redes de comunicação e pela tentativa de controlo da situação (Putman \& Wilson, 1982).

$\mathrm{O}$ evitamento traduz uma baixa preocupação pelos interesses de ambas as partes. A adopção de uma atitude de fuga e de negação do problema existente, característica desta estratégia, mantém o conflito em estado latente, podendo gerar, a longo prazo, maior intensidade conflitual ${ }^{6}$.

Os estudos empíricos realizados na área das estratégias de abordagem aos conflitos têm verificado que a utilização de uma abordagem integrativa revela benefícios para o grupo, quer ao nível do desempenho quer ao nível da satisfação e bem-estar dos seus membros (De Dreu, Dierendonck \& Dijkstra, 2004). Com efeito, a exposição das ideias individuais, estimulada pela utilização de uma estratégia integrativa, facilita a emergência de decisões mais eficazes que traduzem um ganho conjunto, revelando-se benéfica para o grupo, quer no momento da interacção, quer a longo prazo (Alper, Tjosvold \& Law, 2000; Kuhn \& Poole, 2000).

Neste sentido, predizemos que:

Hipótese 2: A utilização de estratégias integrativas tem um impacto positivo no desempenho das equipas de trabalho.

Hipótese 3: A utilização de estratégias integrativas modera a relação entre o conflito grupal e o desempenho.

A utilização de estratégias de domínio, pelo contrário, está associada a baixos níveis de eficácia e de satisfação grupal (Fischer \& Ury, 1981; Friedman, Tidd, Currall \& Tsai, 2000; Pneuman \& Bruehl, 1982). A persistência para alcançar os objectivos individuais intensifica os conflitos existentes, diminuindo as possibilidades de as partes envolvidas chegarem a um acordo (Pruitt \& Carnevale, 1993).

Desta forma, predizemos que:

Hipótese 4: A utilização de estratégias de domínio tem um impacto negativo ao nível do desempenho das equipas de trabalho.

\footnotetext{
${ }^{6}$ Algumas tipologias incluem uma outra estratégia - o compromisso - que descreve uma moderada preocupação com os interesses individuais e com os interesses da outra parte. $\mathrm{Na}$ linha de autores como Pruitt (1983), consideramos, no entanto, que o compromisso não é uma estratégia distinta da integração.
} 
Embora existam algumas evidências que sustentam o impacto negativo da utilização de estratégias não confrontativas (acomodação e evitamento) nos resultados das equipas de trabalho (DeChurch \& Marks, 2001), são poucos os estudos que analisam este tipo de abordagem. Neste sentido, colocamos a seguinte questão exploratória: de que forma a acomodação e o evitamento se relacionam com o desempenho grupal?

\section{Conflitos e desenvolvimento grupal}

O grupo constitui uma realidade dinâmica e mutável que adquire contornos diferentes ao longo da sua existência. No seu processo históricoevolutivo, transforma-se e desenvolve diferentes formas de operar e de se adaptar aos seus contextos (Sundstrom, De Meuse \& Futrell, 1990). Desde os trabalhos de Bales (1950) em torno dos padrões de interacção dos pequenos grupos que se têm multiplicado investigações, teorias e modelos dedicados à evolução dos grupos. Geralmente designados como Modelos de Desenvolvimento dos Grupos, procuram explicar e caracterizar o funcionamento dos mesmos, acentuando a sua dinâmica evolutiva, ao longo do tempo, e enfatizando características específicas de cada nível de existência grupal. Chamando a atenção para o facto de um grupo ser, simultaneamente, diferente e igual a si próprio - diferente porque em contínua umdança, mas igual pela criação e manutenção de uma certa estabilidade estrutural e cultural que lhe confere e "perpetua" uma identidade distintiva -, os referidos modelos apelam para as vantagens de estudar os grupos e seus processos, no seio de uma abordagem que tenha em conta o desenvolvimento grupal.

O estudo das relações entre o conflito e o desenvolvimento grupal, que efectuámos na presente investigação, insere-se na perspectiva que acabámos de enunciar e na linha proposta por Jehn e Mannix (2001), para quem a abordagem aos conflitos deve ser contextualizada no momento temporal em que o grupo se encontra. Nele, procurámos investigar em que medida os diferentes níveis de existência grupal diferem, no que diz respeito, a) à intensidade conflitual, b) ao tipo de conflitos emergentes (tarefa ou sócio-afectivo) e c) às estratégias de abordagem utilizadas na sua gestão (integração, acomodação, domínio e evitamento). Possibilitar uma melhor compréensão do conflito no contexto dos grupos e, simultaneamente, contribuir para testar e enriquecer os modelos de desenvolvimento grupal constituíram, assim, objectivos centrais da investigação que realizámos. Para a sua prossecução, bem como para a fundamentação das hipóteses de investigação formuladas, ancorámo-nos no Modelo Integrado de Desenvovimento Grupal, de Miguez e Lourenço (2001). Trata-se de um modelo que se insere numa perspectiva sociotécnica, sofre influências da Teoria de Campo de Lewin (1951) e é inspirado quer nos modelos lineares (e.g., Modelo Integra- 
do de Desenvolvimento de Wheelan, 1990, 1994), quer nos modelos cíclicos (e.g., Modelo do Grupo Optimal de St. Arnaud, 1978)

De acordo com o modelo de Miguez e Lourenço (2001), o grupo constitui uma "realidade" intersubjectiva que opera em torno de dois subsistemas fundadores - sócio-afectivo e de tarefa - e emerge em função da présença de um conjunto de condições - forças impulsoras de base - que se revelam necessárias e suficientes para a sua génese: percepção de, pelo menos, um alvo comum mobilizador, interdependência e relações entre as péssoas em função do alvo comum.

Enfatizando a dinâmica de um grupo em torno da interdependência dos seus dois subsistemas fundadores, Miguez e Lourenço (2001) concebem, no seu modelo, o desenvolvimento de um grupo até à maturidade, como uma sucessão de quatro estádios - Estruturação, Reenquadramento, Reestruturação e Realização. O carácter cíclico do modelo proposto acentua, no entanto, que o processo de desenvolvimento se faz, muitas vezes, através de avanços e recuos, podendo um grupo fixar-se numa fase particular e aí permanecer indefinidamente. Assim, a maturidade, além de nem sempre ocorrer, assume um carácter transitório.

No primeiro momento de vida do grupo, porque a situação é nova e indefinida, o clima é marcado por uma certa ansiedade. Os membros procuram a sua inclusão no grupo e desenvolvem esforços para agradar ao líder e aos outros membros. Seguem o líder, de quem estão muito dependentes, e procuram conhecer-se uns aos outros através de uma exploração inicial, feita com muito cuidado e de forma defensiva. O receio de ser excluído é, neste período, muito elevado. Por ser sentido como uma ameaça ao equilíbrio e harmonia do grupo, o conflito tende a ser evitado.

\footnotetext{
${ }^{7}$ De acordo com Bouwen e Fry (1996) e também Wheelan e Kaeser (1997), citados por Lourenço (2002), é possível, a este respeito, distinguir quatro tipos de modelos: lineares, cíclicos, de polaridade e de equilíbrio interrompido. Nos modelos lineares, que dominam grande parte da literatura, o desenvolvimento dos grupos é concebido como uma sucessão ordenada de fases ou estádios consecutivos, seguindo uma sequência pré-definida em direcção à maturidade. Os modelos cíclicos concebem o desenvolvimento como um processo circular. A resolução de certos tópicos do grupo é sempre temporária, na medida em que o grupo, evoluindo através de movimentos em espiral, irá, noutro ponto do tempo, retomá-los. Nesta perspectiva, a maturidade grupal será, assim, igualmente, transitória ou sempre em construção, pois o grupo carece de permanente (re)actualização. Nos modelos de polaridade, o desenvolvimento dos grupos é concebido como um contínuo campo de tensões entre forças que se opõem ou equilibram (por exemplo, individualidade/grupalidade). O grupo situar-se-á, em cada momento da sua história, algures num continuum entre as forças em oposição. Para os modelos de equilíbrio interrompido, o desenvolvimento é concebido principalmente em torno da consciência que os membros do grupo possuem relativamente à dimensão temporal, alternando períodos de continuidade, estabilidade e inércia, com períodos de transição e revolução.
} 
No segundo estádio de desenvolvimento do grupo, os membros procuram libertar-se da dependência face ao líder e afirmar a sua individualidade. As diferenças de personalidade e de valores, bem como a presença de perspectivas distintas quanto aos objectivos da equipa e quanto à forma de os alcançar são fonte de tensão e de discórdia. Vive-se um clima de elevada intensidade conflitual, em que os membros do grupo procuram impor-se face aos outros.

Desenvolvimento da confiança, envolvimento em relação ao grupo e incremento do desejo de cooperar marcam o terceiro estádio de desenvolvimento dos grupos, centrado, sobretudo, no sistema-tarefa. É crescente a percepção de interdependência por parte dos membros da equipa, bem como o esforço de aceitação e integração das diferenças. O grupo enceta estratégias mais maduras de negociação relativamente aos objectivos, papéis, estrutura, organização, procedimentos e divisão do trabalho. É esperado que as divergências que surgem conduzam a uma menor intensidade conflitual, sendo geridas, essencialmente, com recurso a estratégias integrativas.

No quarto estádio de desenvolvimento, o grupo canaliza a maior parte da sua energia para a realização das tarefas e para a prossecução dos objectivos partilhados. A percepção de interdependência entre os membros da equipa é clara e valorizada. Vive-se um clima de elevada confiança, envolvimento e cooperação. A comunicação é profunda e facilita a realização das actividades da equipa. Os aspectos que mais caracterizam este estádio são, por isso, os que se prendem com a adequada realização do trabalho ou execução da tarefa e com o reforço da coesão. A diversidade é vivida como uma mais-valia do grupo, razão pela qual a intensidade conflitual tenderá a ser baixa e a utilização de estratégias integrativas elevada.

Tendo em conta as assunções do modelo de desenvolvimento que acabámos de apresentar, formulamos as seguintes hipóteses de investigação:

Hipótese 5: Existem diferenças significativas entre as fases de desenvolvimento, quanto à emergência de conflitos grupais: os conflitos afectivos e de tarefa são mais intensos no estádio 2 (Hipótese 5a); o estádio 1 é o menos conflitual, tanto ao nível sócio-afectivo como ao nível da tarefa (Hipótese 5b).

Hipótese 6: Nos grupos em fases de maior desenvolvimento, a utilização de estratégias integrativas é mais frequente do que nos grupos em fases iniciais do seu desenvolvimento.

Hipótese 7: As estratégias de domínio são mais frequentes na fase 2 do que em qualquer outra fase. 
Hipótese 8: A utilização de estratégias não confrontativas (evitamento e acomodação) é mais frequente nas primeiras etapas do desenvolvimento grupal do que nas etapas finais.

\section{Método}

Amostra

Sessenta e oito equipas de trabalho do departamento de produção de oito organizações do sector industrial participaram no presente estudo. Foram entregues 437 questionários, dos quais 415 foram devolvidos, tendo sido $36(8,7 \%)$ eliminados da amostra, por conterem um número de itens não respondido igual ou superior a 10\% (Bryman \& Cramer, 1990/1993). Após estes procedimentos, a amostra ficou com um total de 379 sujeitos integrados em 64 equipas de trabalho.

As equipas eram compostas por uma média de 9 elementos (D.P. $=$ $5,97)$ e todas desempenhavam tarefas com baixos níveis de complexidade e de carácter rotineiro. Os membros das equipas foram questionados acerca da intensidade e frequência dos conflitos vividos no grupo, das estratégias utilizadas na sua gestão e do nível de desenvolvimento grupal. O desempenho da equipa foi obtido através da avaliação feita pela chefia.

\section{Instrumentos}

Tipo de conflito

Para medir o tipo de conflito, desenvolvemos a Escala de Avaliação do Conflito Intragrupal (EACI), tendo como base alguns instrumentos presentes na literatura da especialidade (Cox, 1998; De Dreu \& Van Vianen, 2001; Jehn, 1994). A EACI é constituída por duas sub-escalas, compostas por 9 itens cada uma - 5 itens avaliam o conflito de tarefa e 4 itens avaliam o conflito afectivo. Na primeira sub-escala avalia-se a frequência com que cada uma das situações apresentadas acontece no grupo $(1=$ nunca acontece; 7 = acontece sempre $)$; na segunda, a tensão por elas gerada $(1=$ nenhuma tensão; $7=$ muita tensão $)^{8}$. Depois de verificarmos a existência de correlações muito elevadas entre as variáveis frequência e intensidade dos conflitos grupais $(r>0,80)$, concluímos que as sub-escalas forneciam informações redundantes, pelo que optámos por utilizar apenas a sub-escala que avalia a intensidade gerada pelas situações conflituais.

\footnotetext{
${ }^{8}$ Exemplos de itens da EACI são: "Ideias diferentes relativamente às regras e aos objectivos da equipa" (conflito de tarefa) e "Divergências entre os membros do grupo associadas a diferenças de personalidade" (conflito afectivo).
} 
Estratégias de gestão de conflitos

Para avaliar as estratégias de gestão de conflitos utilizadas pelos membros do grupo, realizámos uma adaptação ao português do ROCI-II (Rahim, 1983). Este instrumento, na sua versão original, é composto por 28 itens, que medem cinco dimensões que traduzem diferentes formas de agir perante situações conflituais: integração, domínio, evitamento, acomodação e compromisso ${ }^{9}$. Na versão final, resultante dos estudos por nós realizados no processo de adaptação, o ROCI-II integra 15 itens (4 relativos à dimensão integração, 4 ao domínio, 3 ao evitamento e 4 à acomodação) avaliados pelos membros das equipas numa escala de Likert de 7 pontos, que varia entre "nunca acontece" (1) e "acontece sempre" (7)

\section{Desenvolvimento da equipa}

Para avaliar a variável "desenvolvimento" das equipas, utilizámos o PDE (Miguez \& Lourenço, 2001) - Percepção de Desenvolvimento das Equipas. O PDE é constituído por quatro cenários que descrevem as quatro fases do desenvolvimento grupal. Os membros das equipas têm que seleccionar o cenário que melhor descreve o momento actual da equipa.

\section{Desempenho}

O desempenho grupal foi medido através de uma escala de oito itens que avalia diferentes aspectos relativos à qualidade e à quantidade de trabalho produzido pelas equipas. Os itens são avaliados pelos líderes numa escala de 10 pontos $(1=\text { mau; } 10=\text { excelente })^{11}$.

\section{Resultados}

As respostas dadas pelos membros das equipas quanto aos conflitos percepcionados e quanto à fase de desenvolvimento foram agregadas ao

\footnotetext{
${ }^{9}$ Dos 28 itens, quatro avaliam a dimensão compromisso. Visto que, pelas razões já apontadas noutro ponto deste trabalho, não incluímos esta estratégia nas nossas análises, os estudos de adaptação do instrumento foram realizados com base em 24 itens.

10 Um exemplo de item para a integração é "Procuro que os assuntos sejam abordados com frontalidade para que as questões que surgem possam ser resolvidas da melhor forma possível"; "Utilizo o meu poder para ganhar", "Evito o confronto directo com os membros do meu grupo" e "Acomodo-me às aspirações dos outros membros" constituem exemplos de itens que integram, respectivamente, as dimensões domínio, evitamento e acomodação.

11 Alguns exemplos de itens são: "Cumprimento dos níveis de produção exigidos", "Empenho na produção de trabalho de qualidade", "Apresentação de sugestões".
} 
nível grupal ${ }^{12}$. A agregação dos dados permite reduzir o impacto das diferenças individuais dentro de cada equipa, conseguindo-se, desta forma, obter indicadores mais representativos das características dos grupos (Simons \& Peterson, 2000).

Para justificar a agregação dos dados, calculámos o Índice $A D$ (Average Deviation), desenvolvido recentemente por Burke e Dunlap (2002). De acordo com a recomendação dos autores referidos, todos os grupos que não atingiram, naquele índice, valores inferiores a c/6 (sendo c o número de respostas possíveis por item), foram retirados da nossa análise. A amostra final ficou constituída por 50 grupos.

Na tabela 1, são apresentadas as médias, desvios-padrão e inter-correlações para todas as variáveis, bem como a fiabilidade das escalas.

Tabela 1 - Estatísticas Descritivas e inter-correlações para todas as variáveis em estudo.

\begin{tabular}{|c|c|c|c|c|c|c|c|c|c|c|}
\hline Variáveis & $M$ & $D P$ & $\boldsymbol{\alpha}$ & 1 & 2 & 3 & 4 & 5 & 6 & 7 \\
\hline 1. Conflito de tarefa & 2,83 & 0,80 & 0,87 & & & & & & & \\
\hline 2. Conflito afectivo & 2,66 & 0,75 & 0,83 & $0 ., 77 * *$ & & & & & & \\
\hline 3. Desenvolvimento Grupal & 2,92 & 0,86 & - & $-0,27$ & $-0,17$ & & & & & \\
\hline 4. Integração & 4,87 & 0,57 & 0,84 & 0,23 & 0,00 & $-0,05$ & & & & \\
\hline 5. Evitamento & 3,74 & 0,65 & 0,65 & 0,12 & $-0,10$ & $-0,00$ & $0,44 * *$ & & & \\
\hline 6. Acomodação & 3,48 & 0,46 & 0,63 & $0,48 * *$ & 0,23 & $-0,22$ & $0,38 * *$ & $0,45^{* *}$ & & \\
\hline 7. Domínio & 2,42 & 0,62 & 0,70 & $0,42 * *$ & $0,29 * *$ & $-0,06$ & 0,27 & $0,38 * *$ & $0,40 * *$ & \\
\hline 8. Desempenho & 7,39 & 1,14 & 0,88 & $-0,50 * *$ & $-0,44 * *$ & 0,16 & 0,25 & 0,15 & $-0,09$ & $-0,27$ \\
\hline
\end{tabular}

Nota: $* \mathrm{p}<0,05 ; * * \mathrm{p}<0,01$

12 Como a escala das estratégias de gestão de conflitos mede a forma individual de reacção às situações conflituais, não se justificava o cálculo de um índice de acordo. Estes dados foram analisados, posteriormente, calculando a média das estratégias utilizadas pelos membros do grupo, indicador que nos permite conhecer quais as formas de abordagem aos conflitos mais e menos utilizadas pelos membros de cada equipa. 
As escalas utilizadas apresentam valores aceitáveis de fiabilidade ${ }^{13}$. O conflito de tarefa correlaciona-se positivamente com o conflito afectivo e a relação de ambos com o desempenho grupal é negativa, de acordo com o previsto. A utilização de estratégias de acomodação relaciona-se positivamente com o conflito de tarefa e a utilização de estratégias de domínio relaciona-se positivamente com a intensidade conflitual vivida no grupo, quer ao nível da tarefa, quer ao nível afectivo. A relação positiva entre as estratégias integrativas e o desempenho grupal encontra-se perto da significância estatística $(r=0,25, p<0,10)$, tal como a relação negativa entre as estratégias de domínio e o desempenho $(r=0,27, p<0,10)$.

Para testar as hipóteses 1 a 4, conduzimos uma análise da regressão hierárquica com o desempenho grupal como variável dependente (Tabela 2). No primeiro passo introduzimos os dois tipos de conflito, no segundo passo as estratégias de gestão de conflitos e, no terceiro passo, os termos que comtêm as interacções. Assim, o teste à hipótese 1 foi realizado pela análise do primeiro passo e das hipóteses 2 e 4 pela análise do segundo passo. $\mathrm{O}$ efeito moderador da utilização de estratégias integrativas na relação entre o tipo de conflito e o desempenho grupal (hipótese 3 ) foi analisado no terceiro passo.

Com o objectivo de corrigir os problemas de multicolinearidade que podem emergir quando se analisam relações de moderação, as variáveis independentes foram centradas, ou seja, a cada variável independente foi subtraída a média da amostra, seguindo um procedimento proposto por Cohen, Cohen, Aiken e West (2003). Os termos que contêm a interacção foram calculados multiplicando as variáveis centradas.

A análise do primeiro passo indica-nos que, conjuntamente, o conflito de tarefa e o conflito afectivo explicam $22 \%$ da variância da variável dependente. A relação entre o conflito de tarefa e o desempenho é negativa e estatisticamente significativa $(\beta=-0,40, p<0,05)$; no entanto, o efeito negativo do conflito afectivo no desempenho não é significativo $(\beta=-0,13$, n.s.), pelo que foi encontrado apenas um suporte parcial para a hipótese 1. Depois de controlarmos o efeito do conflito grupal, a adição das estratégias de gestão de conflitos, no segundo passo, acrescenta variância significativa no desempenho grupal $(\Delta R 2=0,17, p<0,05)$, apresentando a estratégia integrãtiva um efeito significativo na explicação desta variável $(\beta=0,36$, $p<0,01)$. Encontrámos, desta forma, sustentação empírica para a hipótese 2. A relação entre as estratégias de domínio e o desempenho grupal é negativa, como o previsto, e quase significativa $(\beta=-0,21$, n.s.). Relativamente à nossa questão exploratória, verificámos que não existe qualquer relação

13 Ao contrário de Nunally (1978), que refere que o valor do Alpha de Cronbach deve ser superior a 0,70, Peterson (1994) e também Slater (1995) sugerem que 0,60 é o critério em uso. 
entre a utilização de estratégias não confrontativas (acomodação e evitamento) e o desempenho. A análise, no terceiro passo, dos termos que contêm as interacções, revela-nos que a utilização de estratégias integrativas não modera a relação entre o conflito grupal e o desempenho.

Tabela 2 - Resultados da análise da regressão hierárquica predizendo o desempenho grupal.

\begin{tabular}{|c|c|c|c|}
\hline \multirow[b]{2}{*}{ Variáveis } & \multicolumn{3}{|c|}{ Desempenho Grupal } \\
\hline & $\beta$ & $R^{2}$ Ajustado & $\Delta \boldsymbol{R}^{2}$ \\
\hline 1. & & $0,22 * *$ & $0,26^{* *}$ \\
\hline Conflito de Tarefa & $-0,40^{*}$ & & \\
\hline Conflito Afectivo & $-0,13$ & & \\
\hline 2. & & $0,34 * *$ & $0,17^{*}$ \\
\hline Conflito de Tarefa & $-0,61 * *$ & & \\
\hline Conflito Afectivo & 0,09 & & \\
\hline Integração & $0,36^{* *}$ & & \\
\hline Evitamento & 0,13 & & \\
\hline Acomodação & 0,06 & & \\
\hline Domínio & $-0,21$ & & \\
\hline 3. & & $0,32 * *$ & 0,01 \\
\hline Conflito de Tarefa & $-0,47$ & & \\
\hline Conflito Afectivo & $-0,02$ & & \\
\hline Integração & $0,39 *$ & & \\
\hline Evitamento & 0,12 & & \\
\hline Acomodação & 0,08 & & \\
\hline Domínio & $-0,18$ & & \\
\hline Conflito de tarefa X Integração & $-0,18$ & & \\
\hline Conflito Afectivo X Integração & 0,10 & & \\
\hline
\end{tabular}

Nota: $* p<0,05 ; * * p<0,01$ 
Tabela 3 - Médias do conflito grupal em função da fase de desenvolvimento

\begin{tabular}{lcrrrrc}
\hline & Fase 1 & Fase 2 & Fase 3 & Fase 4 & $\boldsymbol{F ( 3 , 4 6 )}$ & $\boldsymbol{\eta} \mathbf{2}$ \\
\hline Conflito Sócio-afectivo & 2,07 & 3,53 & 2,60 & 2,41 & $6,70 * *$ & 0,30 \\
Conflito de Tarefa & 2,97 & 3,57 & 2,65 & 2,67 & $3,33 *$ & 0,18 \\
\hline Nota: $* p<.0,05 ; * * p<0,01$ & & & & & &
\end{tabular}

Para pôr à prova as hipóteses $5 \mathrm{a}$ e $5 \mathrm{~b}$, realizámos duas ANOVAs, nas quais se introduziu como variável preditora o desenvolvimento grupal e como variáveis critério, o conflito afectivo e o conflito de tarefa. Foram encontradas diferenças significativas entre os níveis de desenvolvimento grupal relativamente à incidência de conflitos afectivos $[F(3,46)=6,7, p<0,01$; $\left.\eta^{2}=0,30\right]$ e de conflitos de tarefa $\left[F(3,46)=3,33, p<0,05 ; \eta^{2}=0,18\right]$.

Tabela 4 - Comparações múltiplas entre as fases de desenvolvimento em função do conflito afectivo

\begin{tabular}{lcccc}
\hline & \multicolumn{4}{c}{ Variável Dependente: Conflito Afectivo } \\
\hline Fase 1 & Fase 1 & Fase 2 & Fase 3 & Fase 4 \\
\cline { 2 - 5 } Fase 2 & & $-1,46^{*}$ & $-0,53$ & $-0,33$ \\
Fase 3 & $1,46^{*}$ & & $0,93^{*}$ & $1,13^{*}$ \\
Fase 4 & 0,53 & $-0,93^{*}$ & & 0,19 \\
\hline
\end{tabular}

Nota: * Médias significativamente diferentes entre si para $p<0,05$, de acordo com o teste de Tuckey

Tabela 5 - Comparações múltiplas entre as fases de desenvolvimento em função do conflito de tarefa

\begin{tabular}{lcccc}
\hline & \multicolumn{4}{c}{ Variável Dependente: Conflito de Tarefa } \\
\hline Fase 1 & Fase 1 & Fase 2 & Fase 3 & Fase 4 \\
\cline { 2 - 5 } Fase 2 & & $-0,60$ & 0,32 & 0,29 \\
Fase 3 & 0,60 & & $0,92^{*}$ & $0,90^{*}$ \\
Fase 4 & $-0,32$ & $-0,92^{*}$ & & $-0,02$ \\
\hline
\end{tabular}

Nota: * Médias significativamente diferentes entre si para $p<0,05$, de acordo com o teste de Tuckey 
A análise das tabelas 4 e 5 (teste post-hoc de Tuckey) permite-nos verificar que, tal como o previsto na hipótese $5 \mathrm{a}$, a fase 2 do desenvolvimento grupal é a mais conflitual, tanto ao nível afectivo, como ao nível da tarefa. É de salientar, no entanto, que não se verifica uma diferença significativa entre a fase 2 e a fase 1 , no que diz respeito aos conflitos de tarefa. Com efeito e contrariamente ao previsto na hipótese $5 \mathrm{~b}$, a intensidade dos conflitos centrados nas tarefas não é significativamente menor na fase 1 do que nas restantes fases do desenvolvimento grupal. Quanto aos conflitos centrados nas relações, são menos intensos na fase 1 do que nas outras fases, sendo, no entanto, esta diferença significativa apenas em relação à fase 2.

Tabela 6 - Médias das estratégias de gestão de conflitos em função da fase de desenvolvimento

\begin{tabular}{lcccccc}
\hline & Fase 1 & Fase 2 & Fase 3 & Fase 4 & $\boldsymbol{F ( 3 , 4 6 )}$ & $\boldsymbol{\eta 2}$ \\
\hline Integração & 5,15 & 4,63 & 4,94 & 4,79 & 1,00 & 0,06 \\
Evitamento & 3,93 & 3,81 & 3,60 & 3,90 & 0,74 & 0,04 \\
Acomodação & 3,82 & 3,68 & 3,35 & 3,49 & 2,07 & 0,12 \\
Domínio & 2,32 & 2,56 & 2,33 & 2,56 & 0,54 & 0,03 \\
\hline
\end{tabular}

Em seguida, para testar as hipóteses 6 a 8, realizámos quatro ANOVAs, nas quais se introduziu como variável preditora o desenvolvimento grupal e como variáveis-critério cada uma das estratégias de gestão de conflitos (cf. Tabela 6). Não existem diferenças significativas entre as 4 fases de desenvolvimento grupal, quanto à utilização das estratégias integrativas $\left[F(3,46)=1,00\right.$, n.s.; $\left.\eta^{2}=0,06\right]$, de evitamento $[F(3,46)=0,74$, n.s.; $\left.\eta^{2}=0,04\right]$, de acomodação $\left[F(3,46)=2,07\right.$, n.s.; $\left.\eta^{2}=0,12\right]$ e de domínio $\left[F(3,46)=0,54\right.$, n.s.; $\left.\eta^{2}=0,03\right]$.

\section{Discussão dos Resultados}

O presente estudo permite-nos compreender melhor as relações que o conflito e as estratégias de gestão de conflitos utilizadas pelos membros do grupo estabelecem com o desempenho grupal.

Contrariando as abordagens que sublinham o impacto positivo dos conflitos centrados na tarefa ao nível da produtividade, criatividade e inovação das equipas de trabalho, o nosso estudo revelou um efeito negativo, estatisticamente significativo, do conflito de tarefa no desempenho, reve- 
lando-se, deste modo, convergente com investigações recentes (a que nos referimos na introdução do presente trabalho). O facto de a nossa amostra ser constituída por grupos que desempenham tarefas com um índice de variabilidade baixo, cuja execução é estandardizada e produz resultados previsíveis, poderá contribuir para explicar a relação negativa encontrada. Com efeito, neste tipo de equipas, a emergência de conflitos de tarefa, ao desviar a energia do grupo da execução da mesma para a resolução de problemas, pode não trazer ganhos para a eficácia, porque os métodos e os procedimentos a executar, além de rotineiros e pouco complexos, são altamente estandardizados. Em grupos que desempenham tarefas de elevada complexidade, em áreas em que os resultados são pouco previsíveis e dependem, em grande escala, da qualidade dos projectos desenvolvidos e dos métodos seleccionados, o impacto do conflito de tarefa ao nível da eficácia poderá ser, a longo prazo, positivo. A realização de um estudo longitudinal com equipas que desenvolvam tarefas com elevado nível de complexidade contribuiria para uma melhor e mais profunda compreensão da relação entre o conflito de tarefa e o desempenho grupal.

Ao contrário do que tinha sido previsto, o conflito afectivo não se revelou um preditor do desempenho grupal. Estes resultados são semelhantes aos obtidos por Jehn (1995) e também por Passos e Caetano (2005), que não encontraram, nos seus estudos, um impacto negativo, estatisticamente significativo, do conflito centrado nas relações sobre o nível de eficácia das equipas. Como uma possível explicação para este dado, aqueles autores apontam o facto de os membros das equipas evitarem trabalhar com quem experienciam conflitos, causados por diferenças de personalidade, valores e perspectivas perante a vida. No caso do presente estudo, porque se trata de equipas fixas e permanentes, onde existe uma reduzida autonomia por parte dos seus membros relativamente à opção sobre "com quem preferem trabalhar", a explicação avançada pelos autores acima citados não parece ajustar-se. Em nossa opinião, é o baixo nível de complexidade e o carácter rotineiro das tarefas realizadas que explicam os resultados obtidos. É que, neste tipo de tarefas, onde os níveis de coordenação de esforços e de partilha de ideias são reduzidos, os níveis de desempenho não estão tão dependentes da qualidade das interacções existentes entre os membros do grupo.

No que diz respeito às estratégias de abordagem aos conflitos, utilizadas pelos membros do grupo, a integração parece ser a única com um efeito positivo significativo no desempenho grupal. A procura de uma solução que integre os vários interesses envolvidos e que resulte num ganho conjunto parece ser a única abordagem com impacto positivo, ao nível dos resultados das equipas de trabalho.

Os resultados do estudo empírico conduzido permitem-nos, ainda, contribuir para clarificar a forma como os conflitos são vividos e geridos ao longo dos diferentes momentos temporais do grupo. 
O primeiro momento de vida do grupo é marcado por poucos conflitos afectivos (no grupo, vive-se a fase de "enamoramento"), mas por uma certa frequência de conflitos de tarefa. Com efeito, a indefinição das tarefas, papéis e objectivos gera alguma tensão no grupo, conduzindo à emergência de divergências entre os membros da equipa. O desejo de inclusão e o receio de se expor inibem a emergência das reais diferenças de personalidade, centrando-se os (poucos) conflitos nas tarefas. Verificámos, tal como tínhamos previsto, que a fase dois é o momento de maior intensidade conflitual tanto ao nível sócio-afectivo, como ao nível da tarefa. No segundo estádio de desenvolvimento, os membros desenvolvem esforços para encontrar o seu lugar no grupo e para se libertarem da autoridade do líder. As diferenças de valores e de personalidade entre os membros do grupo, assim como as divergências relativas ao papel de cada um, aos objectivos e decisões, são fontes de tensão e de discórdia, sendo minimizados os pontos de acordo e as semelhanças existentes. As fases três e quatro caracterizam-se por uma diminuição significativa das divergências centradas quer na tarefa, quer no domínio afectivo. A maior confiança existente entre os membros do grupo, por um lado, associada à delimitação do papel de cada membro, bem como dos objectivos e estratégias da equipa, por outro, permitem que as divergências sejam menos frequentes e de menor intensidade.

A análise da gestão de conflitos, ao longo dos níveis de desenvolvimento grupal, permitiu-nos verificar que, em todos os momentos de vida do grupo, a estratégia mais utilizada pelos seus membros é a integração, seguida do evitamento, da acomodação e do domínio. Contrariamente ao prévisto, parece não haver diferenças significativas quanto à frequência de utilização das diferentes estratégias, ao longo do desenvolvimento grupal.

\section{Limitações}

O presente estudo tem um conjunto de limitações que devem ser salientadas. A sua natureza correlacional, que impede o estabelecimento de relações de causalidade, constitui uma primeira limitação. Com efeito, a direcção das relações entre as variáveis em análise pode, na realidade, ser contrária à prevista (é plausível assumir, por exemplo, que desempenhos baixos podem conduzir à emergência de maior intensidade conflitual). $\mathrm{O}$ facto de o estudo ter sido realizado num momento único coloca, por outro lado, problemas à análise do desenvolvimento grupal: os grupos podem ser diferentes entre si ao longo do tempo. Um estudo longitudinal que explorasse a forma como os diferentes processos grupais, nomeadamente o conflito, são vividos por cada grupo ao longo do seu desenvolvimento contribuiria significativamente para o aprofundar de conhecimentos sobre esta problemática. 
Uma outra limitação relaciona-se com a natureza auto-descritiva das medidas, que pode conduzir à ameaça da variância do método comum (common method variance $)^{14}$. Para reduzir o risco desta ameaça, efectuámos dois procedimentos: as variáveis "tipo de conflito" e "desenvolvimento grupal" foram obtidas através de diferentes avaliadores e posteriormente agrupadas; o "desempenho" das equipas foi avaliado pelos líderes (Conway, 2002; Spector, 1987).

Por último, o presente estudo focaliza-se exclusivamente em equipas de trabalho que desempenham tarefas rotineiras e com um grau de complexidade reduzido, o que limita a generalização dos dados para outro tipo de grupos. Em futuras investigações, é fundamental incidir sobre equipas cujas tarefas têm um elevado nível de complexidade, de forma a testar o padrão de resultados encontrado.

\section{Referências}

Alper, A., Tjosvold, D. \& Law, K. S. (2000). Conflict management, efficacy and performance in organizational teams. Personnel Psychology, 53, 625-642.

Amason, A. C. (1996). Distinguishing the effects of funcional and dysfuncional conflict on strategic decision making: resolving a paradox for top management teams. Academy of Management Journal, 39, 123-148.

Amason, A. C. \& Sapienza, H. (1997). The effects of top management team size and interaction norms on cognitive and affective conflict. Journal of Management, 23, 496-516.

Amason, A. C. \& Schweiger, D. M. (1997). The effects of conflict on strategic decision making effectiveness and organizational performance. In C. D. Dreu \& E. Van de Vliert (Eds.), Using conflict in organizations (pp. 101-115). London: Sage Publications.

Bales, R. F. (1950). Interaction process analysis: a method for the study of small groups. Cambridge. Mass: Addison-Wesley.

Blake, R. \& Mouton, J. (1964). The managerial grid. Houston: Gulf.

Bryman, A. \& Cramer, D. (1993). Análise de dados em ciências sociais. Introdução às técnicas usando o SPSS (2. ${ }^{\mathrm{a}}$ ed.). Oeiras: Celta Editora (versão original: 1990).

Burke, M. J. \& Dunlap, J. P. (2002). Estimating interrater agreement with the average deviation index: a user's guide. Organizational Research Methods, 5 , $159-172$

14 A variância do método comum (common method variance) pode ser definida como uma forma de erro sistemático ou de contaminação, causada pelo método utilizado e não pelo traço medido (Conway, 2002). Quando não controlado, este tipo de ameaça pode conduzir à emergência de correlações que não traduzem relações reais entre as variáveis em estudo. 
Cohen, J., Cohen, P., West, S. G. \& Aiken, L. (2003). Applied multiple regression/correlation analysis for the behavioral sciences. London: Lawrence Erlbaum Associates, Publishers.

Conway, J. M. (2002). Method variance and method bas in industrial and organizational psychology. In S. G. Rogelberg (Ed.), Handbook of research methods in industrial and organizational psychology (pp. 344-365). Oxford: Blackwell Publishing, Lda.

Cox, K. B. (1998). Antecedents and effects of intergroup conflict in the nursing unit. unpublished doctoral dissertation, Virginia Commonwealth University, Richmond, VA.

De Dreu, C. K. W., Dierendonck, D. \& Dijkstra, M. T. M. (2004). Conflict at work and individual well-being. International Journal of Conflict Management, 15, 6-26.

De Dreu, C. K. W. \& Van Vianen, A. E. M. (2001). Managing relationship conflict and the effectiveness of organizational teams. Journal of Organizational Behavior, 22, 309-328.

De Dreu, C. K. W. \& Weingart, L. R. (2003a). A contingency theory of task conflict and performance in groups and organizational teams. In M. A. West, D. Tjosvold \& K. G. Smith (Eds.), International handbook of organizational teamwork and cooperative working (pp. 150-166). Chichester: John Wiley \& Sons, Ltd.

De Dreu, C. K. W. \& Weingart, L. R. (2003b). Task versus relationship conflict, team performance, and team member satisfaction: a meta-analysis. Journal of Applied Psychology, 88, 741-749.

DeChurch, L. A. \& Marks, M. A. (2001). Maximizing the benefits of task conflict: the role of conflict management. Journal of Conflict Management, 12, 4-22.

Deutsch, M. (1973). The resolution of conflict. New Haven, CT: Yale University Press.

Dimas, I. D., Lourenço, P. R. \& Miguez, J. (2005). Conflitos e desenvolvimento nos grupos e equipas de trabalho: uma abordagem integrada. Psychologica, 38, 103-119.

Fisher, R. \& Ury, W. (1981). Getting to yes: negotiating agreement without giving in. London: Hutchinson.

Friedman R. A., Tidd S. T., Currall S. C. \& Tsai J. C. (2000). What comes around cames around: the impact of personal conflict style on work conflict and stress. The International Journal of Conflict Management, 11, 32-55.

Guetzkow, H. \& Gyr, J. (1954). An analysis of conflict in decision making groups. Human relations, 7, 367-381.

Guzzo, R. A. \& Shea, G. P. (1992). Group performance and intergroup relations in organizations. In M. Dunnette \& L. M. Hough (Eds.), Handbook of industrial and organizational psychology (pp. 269-313). Palo Alto: Consulting Psychologists Press Inc.

Jehn, K. A. (1994). Enhancing effectiveness: an investigation of advantages and disadvantages of value-based intragroup conflict. International Journal of Conflict Management, 11, 56-73. 
Jehn, K. A. (1995). A multimethod examination of the benefits and detriments of intragroup conflict. Administrative Science Quarterly, 40, 256-282.

Jehn, K. A. \& Mannix, E. (2001). The dynamic nature of conflict: a longitudinal study of intragroup conflict and group performance. Academic of Management Journal, 44, 238-251.

Kuhn, T. \& Poole, M. S. (2000). Do conflict management styles affect group decision making? Evidence form a longitudinal study. Human Communication Research, 26, 558-590.

Lewin, K. (1951). Field theory in social science. New York: Harper.

Lourenço P. R. (2002). Concepções e dimensões da eficácia grupal: desempenho e níveis de desenvolvimento. Dissertação de Doutoramento, Faculdade de Psicologia e de Ciências da Educação, Universidade de Coimbra, Coimbra.

Lovelace, K., Shapiro, D. L. \& Weingart, L. R. (2001). Maximizing crossfunctional new product teams' innovativeness and constraint - adherence: a conflict communications perspective. Academy of Management Journal, 44, 779-793.

Medina, F., Munduate, L., Dorado M., Martínez, I. \& Guerra J. (2005). Types of conflict and affective reactions. Journal of Managerial Psychology, 20, 219-230 .

Miguez, J. \& Lourenço, P. R. (2001). Qual a contribuição da metáfora "equipa" para a abordagem da eficácia organizacional? Comunicação apresentada no âmbito do IV Encontro Luso-Espanhol de Psicologia Social, 9/Nov., Porto, Universidade Lusíada.

Nunally, J. (1978). Psychometric theory. New York: Mc Graw-Hill.

O’Reilly, C. A., Williams K. Y. \& Barsade, S. (1998). Group demography and innovation: does diversity help? In M. A. Neale, E. A. Mannix \& D. H. Gruenfeld (Eds.), Research on managing groups and teams (pp. 183-207). London: Jai Press inc.

Passos, A. \& Caetano, A. (2005). Exploring the effetcs of intragroup conflict and past performance feedback on team effectiveness. Journal of Managerial Psychology, 20, 231-244.

Peterson, R. (1994). A meta-analysis of Cronbach's coefficient alpha. Journal of Consumer Research, 21, 381-391.

Pneuman, R. W. \& Bruehl, M. E. (1982). Managing conflict. Englewood Cliffs, NJ: Prentice-Hall.

Pruitt, D. G. (1983). Strategic choice in negotiation. American Behavioral Scientist, 27, 167-194.

Pruitt, D. G. \& Carnevale, P. J (1993). Negotiation in social conflict. Pacific Groves, CA: Brooks/Cole.

Pruitt, D. G. \& Rubin, J. Z. (1986). Social conflict: escalation, stalemate and settlement. New York: Random House.

Putnam, L. L. (1988). Communication and interpersonal conflict in organizations. Management Communication Quarterly, I, 293-301.

Putman, L. L. (1997). Productive conflict: negociation as implicit coordination. In C. D. Dreu \& E. Van de Vliert (Eds.), Using conflict in organizations. London: Sage Publications. 
Putnam, L. L. \& Wilson, C. E. (1982). Communicative strategies in organizational conflicts: reliability and validity of a measurement scale. In M. Burgoon (Ed.), Communication yearbook (vol. 6, pp. 629-652). Beverly Hills, CA: Sage.

Rahim, M. A. (1983). A measure of styles of handling interpersonal conflict. Academy of Management Journal, 26, 368-376.

Rentsch, J. R. \& Zelno, J. A. (2003). The role of cognition in managing conflict to maximize team effectiveness. In M. A. West, D. Tjosvold \& K. G. Smith (Eds.), International handbook of organizational teamwork and cooperative working (pp. 130-149). Chichester: John Wiley \& Sons, Ltd.

Schweiger, D. \& Sandberg, W. (1989). The utilization of individual capabilities in group approaches to strategic decision making. Strategic Management Journal, 10, 31-43.

Schwenk, C. \& Cosier, R. (1993). Effects of consensus and devil's advocacy on strategic decision making. Journal of Applied Social Psychology, 23, 226-139.

Simons, T. L., Pelled, L. H. \& Smith, K. A. (1999). Making use of difference: diversity, debate and decision comprehensiveness in top management teams. Academy of Management Journal, 42, 662-673.

Simons, T. L. \& Peterson, R. S. (2000). Task conflict and relationship conflict in top management teams: the pivotal role of intragroup trust. Journal of Applied Psychology, 85, 102-111.

Slater, S. (1995). Issues in conducting marketing strategy research. Journal of Strategic Research, 3, 257-270.

Spector, P. E. (1987). Method variance as an artefact in self-report measures of job stressors and strain: myth or significant problems. Journal of Applied Psychology, 72, 438-443.

Spector, P. E. \& Jex, S. M. (1998). Development of four self-report measures of job stressors and strain: interpersonal conflict at work scale, organizational constraints scale, quantitative workload inventory, and physical symptoms inventory. Journal of Occupational Health Psychology, 3, 356-367.

St. Arnaud, Y. (1978). Les petits groupes: participation et communication. Montréal: Les Presses de L'Université de Montréal - Les Editions du CIM.

Sundstrom, E., De Meuse, K. \& Futrell, D. (1990). Work teams: applications and effectiveness. American Psychologist, 45, 120-133.

Thomas, K. (1992). Conflict and negotiation processes in organizations. In M. Dunnette (Ed.), Handbook of industrial and organizational psychology (vol. 3, pp. 651-717). Palo Alto: Consulting Psychologists Press Inc.

Tjosvold, D. (1997). Conflict within interdependence: its value for productivity and individuality. In C. D. Dreu \& E. Van de Vliert (Eds.), Using conflict in organizations (pp. 23-37). London: Sage Publications.

Turner, M. E. \& Pratkanis, A. R. (1994). Social identity maintenance prescriptions for preventing groupthink: reducing identity protection and enhancing intellectual conflict. International Journal of Conflict Management, 5, 254-270 . 
Turner, M. E. \& Pratkanis, A. R. (1997). Mitigating groupthink by stimulating constructive confict. In C. D. Dreu \& E. Van de Vliert (Eds.), Using conflict in organizations (pp. 53-71). London: Sage Publications.

Wheelan, S. (1990). Facilitating training groups: a guide to leadership and verbal intervention skills. New York: Praeger.

Wheelan, S. (1994). Group processes: a developmental perspective. Needham Heights, MA: Allyn \& Bacon. 